Sección I

ENFOQUES 



\title{
La Ciencias Sociales en la Docencia UNIVERSITARIA.
}

\author{
SOCIAL SCIENCES In University TEACHING
}

\author{
Raul Atria Benaprés \\ Especialista en Educación Superior, \\ Estratificación Social y Teoría Sociológica, \\ Universidad de Chile \\ Cap. Ignacio Carrera Pinto \#1045, Nuñoa \\ ratria@uchile.cl
}

Resumen: El artículo destaca los profundos procesos de cambio que acompañan la transformación modernizante de la sociedad contemporánea. Por ello, es imperativo examinar la formación de recursos humanos en el campo de las ciencias sociales para enriquecer la capacidad de intervención social de los jóvenes profesionales de hoy. Al respecto, hay que enfrentar desafíos mayúsculos para asegurar la relevancia de la docencia universitaria, entendida como un proceso transformador del estudiante y dinámico en la entrega de contenidos intelectuales. El pregrado es el primer escenario de esa transformación. La investigación es indispensable en la docencia de posgrado y es altamente conveniente en el pregrado, especialmente en los niveles avanzados de los currículos. Hay tres contribuciones de las ciencias sociales para responder a esos desafíos: desarrollar pensamiento analítico, desarrollar capacidad investigativa y adiestrar en las prácticas profesionales. La docencia universitaria de pregrado debe asumir la innovación curricular como una tarea ineludible en las instituciones de educación superior.

Palabras clave: ciencias sociales, docencia universitaria, transformación, innovación curricular, investigación

Abstract: This article highlights the deep processes of change that go along the modernizing transformation of contemporary society. Therefore, it is imperative to review the formation of human resources in the field of social sciences for improving the social intervention skills of young professionals today. To that end, some major challenges must be faced for assuring the relevance of university teaching when the latter is understood as a process for transforming students, which is also dynamic in delivering its intellectual contents. Undergraduate teaching is the first scenario for this transformation. Research is a fundamental requirement for post graduate teaching and it is highly commendable for undergraduate teaching, particularly at the advanced levels of the curricula. There are three contributions that the social sciences can make for facing those challenges: development of analytical thought, development of research capacities, and training in the practice of the professions. Undergraduate university teaching must assume curricular innovation as a compulsory task for higher education institutions.

Key words: social sciences, university teaching, transformation, curriculum innovation, research 


\section{INTRODUCCIÓN: EL ESCENARIO DE LOS CAMBIOS.}

Desde la década de los años 1980 la sociedad chilena está experimentado profundas transformaciones y cambios que encuentran su raíz en algunos ejes mayores que son: a) la dinámica de cambios en la estructura sociodemográfica que es inducida por el proceso de modernización de la sociedad; b) los efectos socioeconómicos producidos por el modelo de desarrollo que da sustento a las políticas públicas aplicadas en el país desde mediados de los 1970; c) la recepción de los valores y actitudes de la modernidad que influyen en la vida social, las prácticas políticas y los nuevos estilos de vida de los grupos sociales en el país. El efecto combinado de estos tres grandes ejes de la dinámica de cambios (modernización, modelo de desarrollo y modernidad) es un vasto proceso de transformación de escala societal (es decir, que está tocando todos los ámbitos de la sociedad) pero al mismo tiempo heterogéneo (es decir, diversificado y contradictorio por las distintas intensidades con que se desenvuelve). Muchos de estos cambios también repercuten en el contexto de América Latina, aunque con particularidades propias de los distintos países de la región.

Con todo, algunas de las manifestaciones sociales y demográficas más significativas de la modernización provienen del avanzado proceso de transición demográfica que está experimentando el país, que se expresa en la baja sostenida de la tasa de natalidad, el aumento de la esperanza de vida, cambios en las causas de muerte, envejecimiento de la población, los cambios en las relaciones de dependencia y en la composición de la población económicamente activa, y la disminución del crecimiento natural de la población. Asimismo, el avanzado proceso de urbanización ha cambiado los patrones de distribución territorial del país que fortalece de manera notable el crecimiento de sus ciudades intermedias, acarreando también modificaciones al patrón de desarrollo territorial y regional, y cambios duraderos en la composición sectorial de la estructura ocupacional y del empleo.

El modelo de desarrollo, sustentado en la racionalidad de los incentivos del mercado para la asignación de recursos, (desregulación para maximizar la apertura a la iniciativa privada, gestión autónoma de la política monetaria, y reasignación del gasto público), con sostenida apertura hacia la economía global para incentivar la competitividad de las empresas, y la privatización de servicios públicos, ha generado una profunda alteración de las tareas del Estado y una persistente y urgente necesidad de redefinir su rol en la economía y en la sociedad. El Estado es hoy una entidad más heterogénea, y contradictoria, es decir altamente tecnificado y eficiente en algunos de sus ámbitos y esferas de 
acción, pero al mismo tiempo defectuosamente burocratizado en otras, y con muy disímiles competencias regulatorias en diversos sectores.

Las prácticas, valores y actitudes propias de la modernidad cultural que enfatizan la auto referencia y la auto determinación del sujeto individual, están teniendo una progresiva difusión en la sociedad chilena en general, pero especialmente en los estratos medios y en los grupos técnicos y profesionales compuestos por jóvenes y adultos jóvenes. Esto lleva a cambios muy profundos en las instituciones sociales que tienen más incidencia en la vida cotidiana, como son por ejemplo las familias, los grupos vecinales, los movimientos de base religiosa. El tejido social de la identidad, de la afiliación política y de la participación ciudadana está experimentando transformaciones también profundas. Si no se captan estas nuevas formas de pertenencia y de participación se tiende a pensar que hay en los grupos ya indicados una apariencia de retraimiento contestatario que desconcierta a los actores sociales más tradicionales. Lo que ocurre sin embargo es que las formas de pertenencia, de identidad y de involucramiento de las personas en la vida social y política del país tienen ahora formas de expresión que no son plenamente canalizadas por las organizaciones establecidas, tales como los partidos, las organizaciones sociales de base, los sindicatos. De este modo lo que hay es una sociedad muy dinámica pero con una importante cuota de desarraigo.

El efecto combinado de estos ejes de cambios introduce una compleja configuración donde hay conciencia de logros pero al mismo tiempo una aguda insatisfacción y una carencia del sentido hacia el cual está evolucionando la sociedad. En estas condiciones surge entonces una forma de actuar y de relacionarse con los demás que es muy ajena a las formas convencionales o tradicionales de comportamiento. Esto se observa de manera claramente perceptible en la esfera de la política, pero no sólo en ella. Se trata de un rasgo mucho más generalizado, que también afecta al ámbito de la economía y el trabajo, a las relaciones domésticas y familiares, a las relaciones intergeneracionales y también, de manera especial, a la educación superior universitaria.

\section{LA DOCENCIA UNIVERSITARIA}

Como resultado de los amplios y a la vez profundo procesos de cambio que se observan en las sociedades hoy expuestas a la trasformación modernizante, todas las prácticas sociales se ven afectadas por el contexto de los cambios. Desde un punto de vista de las acciones para actuar en ese escenario dinámico 
desde la perspectiva de la educación superior, es especialmente importante examinar con cierta detención lo que ocurre con la formación de recursos humanos altamente calificados en el campo de las disciplinas de las ciencias sociales, que tienen que enriquecer la capacidad de intervención social de los jóvenes profesionales especializados en estas disciplinas. Ello obliga a plantear algunos temas centrales relacionados con las ciencias sociales y su relación con la docencia en el actual contexto de la educación superior en el país.

Podemos convenir en que la docencia es el proceso interactivo mediante el cual un estudiante recibe programadamente un conglomerado de información en un determinado nivel de alcance y complejidad y aprende a efectuar transformaciones con ese conglomerado para acceder a un nivel cualitativamente superior. Las transformaciones se logran mediante el acceso a otra información que es normativa, pues contiene reglas y criterios metodológicos de procedimientos para la transformación. El currículo es el proceso que recoge y organiza secuencialmente esta creciente complejidad de la formación universitaria que culmina en la construcción de un sujeto superior como transformación se basa en una noción de cambio cualitativo, en que se entiende la educación no como un servicio a un usuario, sino un proceso continuo de transformación del participante (Harvey y Green, 1997)

Hay dos componentes en este enfoque. Uno es el enriquecimiento del estudiante, pues se entiende que una educación de calidad es aquella que logra transformar a los participantes y que, en concreto, logra aumentar el conocimiento, las habilidades y destrezas de sus estudiantes, pudiendo medirse este logro a través del valor agregado en una perspectiva multidimensional. El otro componente es el empoderamiento del estudiante, es decir, la capacidad de dar poder a los estudiantes para participar en el proceso de aprendizaje y por tanto, en su propia transformación. Aspectos tales como la satisfacción de los estudiantes, la flexibilidad curricular, o el desarrollo del pensamiento crítico son esenciales para generar estos procesos de transformación.

En estos términos, la calidad estaría referida al modo por el cual el sistema educativo transforma la capacidad conceptual y la autoconciencia de los estudiantes y su desarrollo como sujetos autónomos. La participación en decisiones institucionales es un componente importante, que se evidencia en la democratización del proceso y no sólo en la evaluación de los resultados.

La persona que pasa por la universidad no sólo es un sujeto formado en su especialidad, sino un sujeto transformado. La formación puede darse en el ámbito de uno solo de los polos de actividad. Pero la transformación del 
estudiante es siempre el resultado de su exposición a la pluralidad de lenguajes y ello ocurre cuando el currículo lo abre sistemáticamente a esa pluralidad (Barnett, 1994).

El pregrado es el primer escenario de esa transformación que culmina en la autonomía del sujeto (Atria, 2000).

El proceso no es lineal, por cuanto el nivel superior de alcance del conglomerado de información no es necesariamente más amplio ni más estrecho. Puede ser de una u otra forma. Hay en efecto, encadenamientos de contenidos en los cuales se va ampliando el alcance de la información. Se trata de contenidos que tienden a la generalización; hay otros contenidos, sin embargo, que tienden a la focalización. En determinadas líneas curriculares la progresión va hacia la generalización; en otra, en cambio, la progresión va hacia la focalización. Esto significa que la docencia en el marco de un programa, puede tener fases hacia una $u$ otra perspectiva, en secuencias que no son necesariamente coincidentes. Es decir, podemos imaginar una gran plasticidad de la docencia para combinar, dentro de un currículo, encadenamientos de todo tipo. Los puntos críticos estarán entonces en las claves de transformación de conglomerado (paquetes) y tipos (clases) de información.

El carácter activo de la docencia no está tanto en la transmisión y apropiación de contenidos (paquetes y clases de información) sino principalmente en la operación de las claves de transformación. Estas claves se manejan con destrezas generales (saber formular un problema, saber verbalizar, saber hacer operaciones formales, saber descomponer y recombinar elementos de una estructura, saber cómo someter a crítica proposiciones argumentativas).

Si entendemos la docencia de esa manera, es decir como un proceso proactivo respecto del estudiante y dinámico en la entrega de sus contenidos, podemos entonces intentar algunas reflexiones acerca de la relación que está llamada a tener la investigación con la docencia.

Desde el punto de vista del aprendizaje de competencias, en el contexto de las transformaciones arriba indicadas, se configuran demandas de cambio curricular que tienen incidencia directa en la educación superior y que exigen una formación que apunta a mayores y mejores capacidades para abstraer y elaborar conocimientos, para pensar en configuraciones sistémicas, para experimentar y aprender a aprender, para comunicarse y trabajar colaborativamente, para resolver problemas y para, finalmente, manejar incertidumbre aprendida y adaptarse mejor al cambio. El impacto de estas 
demandas provoca agudas tensiones en el proceso formativo que se imparte en la educación superior las cuales se expresan una articulación problemática y difícil en las competencias que se adscriben usualmente a los currículos de las carreras.

Para aclarar estas tensiones que operan desde el seno de los currículos, pueden distinguirse dos grandes ámbitos de competencias: uno que se configura en torno a las competencias operacionales de las profesiones y otro que se arma en torno a las competencias propiamente académicas de las mismas. La tensión entre ambas configuraciones, atraviesa todas las dimensiones del aprendizaje, como se ilustra a continuación.

\section{Cuadro No. 1. Competencias curriculares en la educación superior}

\begin{tabular}{|l|l|l|}
\hline Dimensiones del aprendizaje & Competencias operacionales & Competencias académicas \\
\hline Epistemología & Saber cómo & Saber qué/porqué \\
Definición de situaciones & Pragmáticamente & Por campo intelectual \\
Focalización & Resultados & Proposiciones \\
Comunicación & Estratégica & Códigos disciplinarios \\
Formación & Experimental & Disciplina \\
Evaluación & Eficiencia & Veracidad \\
Límites & Normas organizacionales & Normas disciplinarias \\
\hline
\end{tabular}

Fuente: Cristián Cox,

La importancia de este cuadro para el tema de este trabajo, no está tanto en el señalamiento de la polaridad entre las competencias operacionales que se organizan en torno a destrezas técnicas y las académicas, que se organizan en torno a la internalización de contenidos de significación disciplinaria, sino en la inevitable articulación de ambas en la formación profesional universitaria. Esta exige abordar los dos polos de la profesión, de manera tal que un estudiante que completa el ciclo del pregrado deberá estar equilibradamente pertrechado de competencias tanto operacionales como académicas.

Si se lee el cuadro horizontalmente desde las líneas de la primera columna, aparecen algunas dimensiones que pueden servir de ejes para producir la articulación deseada de los dos polos de las competencias. Esto significa, por ejemplo, que en términos de la dimensión epistemológica de su profesión, un estudiante egresado del plan de estudios de una carrera universitaria, se ha apropiado de un conocimiento que fundamenta el cómo hacer las cosas propias de su profesión y el por qué las hace de esa manera y no de otra. El 
resto de las dimensiones curriculares proporcionan, de la misma manera, las formas precisas en que puede entenderse la articulación de los dos tipos de competencias diferenciadas en el cuadro.

Desde el punto de vista de la formación de post grado, la Universidad es el escenario que se diseña para crear condiciones favorables y sostenidas de desarrollo de la investigación como un campo fundamental para la educación superior. Es un escenario que se sostiene sobre la base de la relación virtuosa que existe entre la capacidad investigativa que radica en el sistema y el logro del desarrollo como tarea nacional.

Los actores principales en ese escenario son aquellas instituciones a las cuales se les reconoce un lugar de reconocida calidad en la cúspide del sistema. Este reconocimiento se funda en el hecho de que ellas son entidades completas en la cobertura de las diversas áreas del conocimiento, que extienden sus actividades académicas, y por ende sus desarrollos disciplinarios lo cual incluye tareas de investigación, hacia todas las áreas del conocer. Se trata de universidades que realizan investigación institucionalizada, de cierta magnitud y cobertura, de modo que estas actividades ya no dependen primordialmente del empeño individual de uno o más académicos que investigan como propietarios interesados del tema o proyecto, sino que involucran efectivamente una masa crítica que se constituye como expresión de una voluntad política y un apoyo institucional expreso y sostenido.

El componente de investigación está presente en todas sus unidades académicas, incluyendo aquellas que no están en el núcleo de las ciencias experimentales sino en las ciencias sociales, en las disciplinas interpretativas y en las áreas expresivas de la creación artística. Los académicos de estas universidades postulan y ganan concursos de investigación en todas las áreas y por ende publican también en todas las áreas. A estas instituciones se las suele englobar bajo la designación de complejas, de modo que para las disciplinas que se alojan en ellas, el teorema básico es que no hay desarrollo teórico disciplinario sin investigación.

Pueden reconocerse actualmente distintas prácticas de investigación al interior de las universidades aparte de la investigación académica disciplinaria que ha sido un quehacer definitorio de la universidad como institución, como es el caso de la investigación de servicio y la investigación institucional. La primera responde a la creciente apertura de la universidad al medio externo que es demandante de investigación aplicada, en tanto la segunda surge como 
respuesta a la presión de los procesos de acreditación que se dirigen a las universidades desde el medio externo.

La investigación disciplinaria se enmarca en ámbitos de pensamiento relativamente delimitados que cuentan con un núcleo de teoría y método construido desde una disciplina fundante. La delimitación de estos ámbitos es a veces una frontera muy definida, pero otras veces consiste de espacios fronterizos abiertos donde emergen problemas u objetos de investigación que desplazan la línea fronteriza de la disciplina hacia espacios conceptuales que de alguna manera están más próximos a otras disciplinas. Por ello es que la investigación tiene facetas de consolidación de la disciplina como de apertura hacia la interdisciplina.

Este tipo de investigación es indispensable en el nivel de la docencia de postgrado de modo que una institución que no realice esta actividad investigativa no está en condiciones de sustentar docencia de este nivel. En la docencia de pregrado esta investigación es altamente conveniente en los niveles más avanzados del currículo de especialización y en las asignaturas de metodología.

La investigación institucional conduce a la producción de conocimiento acerca de las dinámicas internas de la institución y sus interacciones con los cambios en el contexto desde donde provienen las demandas formativas hacia la universidad. Mirada desde la docencia esta investigación es valiosa tanto para el pre como para el postgrado pero es en esta última donde puede ser un área fértil para la realización de las tesis de graduación.

Se puede reconocer también un tipo de investigación que se hace para la docencia, en particular toda aquella que desde las ciencias de la educación, busca producir conocimiento acerca de la validez y efectividad de los currículos y los métodos e aprendizaje. Esta investigación resulta indispensable para sustentar una docencia de pregrado que tenga pertinencia y calidad formativa como se ha señalado más arriba. Hay además un tipo de investigación que se hace como producto de la docencia y que genera, como resultado, la producción de textos, manuales y guías para el desarrollo de determinados contenidos curriculares.

Finalmente, la investigación de servicio o aplicada, corresponde a toda aquella actividad de producción de conocimiento que es gatillada por la demanda de un agente externo que requiere del conocimiento experto que puede proporcionar la academia. Las universidades, a veces institucionalmente y también como respuestas más o menos colectivas de grupos de académicos, 
producen investigación de este tipo. Las ciencias sociales han entrado con fuerza en este ámbito de actividad, que solía estar dominado por disciplinas que se caracterizan por tener una estrecha interacción entre las expresiones científicas y tecnológicas de sus ámbitos de conocimiento, como es, por ejemplo, el caso de las ingenierías. La relación más precisa que pueda darse entre este tipo de investigación y la docencia universitaria de pre y post grado, dependerá de la fuerza que tengan las corrientes profesionalizantes en las diversas disciplinas de las ciencias sociales.

Para la docencia de postgrado la investigación es un elemento definitorio, sin embargo, el nexo con la investigación es relativamente complejo. Mientras más se acerca la investigación, de cualquier tipo que ella sea, hacia las fronteras analíticas de las disciplinas, mayor es su directa relación con el componente generalizante de la docencia; mientras mayor es el acercamiento de la investigación hacia la factualidad empírica, más fuerte es su incidencia en el componente de la especialización de la docencia.

El principal desafío para una docencia de post grado de alta calidad, es el logro de una combinación que articule virtuosamente estas dos orientaciones de la investigación.

\section{LA APERTURA HACIA LAS CIENCIAS SOCIALES.}

El área académica de las ciencias sociales constituye un importante espacio que debiera ser profundamente enriquecedor para la Universidad. Esta apertura al vasto campo del conocimiento que se articula en torno a las disciplinas sobre las que se construyen las ciencias sociales modernas, implica necesariamente una toma de contacto y un diálogo con las prácticas de la investigación empírica. Es decir, las ciencias sociales introducen la dimensión del conocimiento factual de la realidad social, aquel espacio de la vida colectiva que se fundamenta en las relaciones entre sujetos humanos, en la interpretación de sentido que estos hacen de esa vida y en las prácticas sociales comunicativas con las cuales los sujetos se constituyen como actores de la realidad social. Hay aquí varias perspectivas que concurren a conformar el objeto de análisis de las ciencias sociales (Atria, 2011)

A manera indicativa se pueden señalar algunas de esas perspectivas, tales como la dimensión interactiva de las redes y estructuras de relaciones en las que se desarrolla la vida colectiva; la construcción de sentido que hacen los sujetos pertrechados con su acervo cultural; las formas de acción mediante 
las cuales los sujetos sociales se constituyen en actores y movimientos que plasman la sociedad de la cual son parte.

Como puede advertirse en este excurso un tanto analítico, lo que está implicado en las ciencias sociales es un proceso de inteligibilidad de la realidad social que es parte indispensable para asumir la sociedad moderna como un proyecto en construcción más que como un orden social preestablecido y garantizado. Ese proceso de inteligibilidad se articula desde disciplinas que están cada vez más relacionadas e interpenetradas, pero sin ellas perder (todavía) el núcleo analítico (es decir el cuerpo teórico y epistemológico) constitutivo que está en sus paradigmas clásicos. Las ciencias sociales modernas entonces están insertas en una vasta matriz conceptual conectada con la factualidad de los fenómenos sociales, en que se distinguen perspectivas para entender los procesos de construcción de sentido, la racionalidad de la acción económica, las relaciones de poder que moldean la acción política, la constitución y reproducción de estructuras de interacción social, la temporalidad histórica de la vida social, los códigos y procesos comunicativos, para mencionar lo que parecen ser los focos disciplinarios más característicos de las ciencias sociales en la actualidad.

Lo dicho hasta aquí permite sostener que este vasto programa multidisciplinario para la inteligibilidad de la sociedad posee la identidad y la densidad suficiente como para constituir de suyo una facultad completa, con todas las facetas definitorias de una actividad académica de nivel superior. Si se pasa revista a las diversas maneras en que se están institucionalizando las ciencias sociales, las humanidades y el pensamiento especulativo, en las universidades hoy día, se constata que hay una gran diversidad de modelos para dar forma a la organización y gestión de esa institucionalización. No hay por consiguiente modelos que tengan superioridad sobre otros cuando se aborda la cuestión de crear el entorno más apropiado, en la estructura universitaria, para incorporar a las ciencias sociales en el proyecto institucional de la universidad de que se trate.

Lo fundamental es cautelar los rasgos propios de este conjunto de disciplinas, que se han mencionado más arriba, es decir: el aprendizaje de las destrezas analíticas de estas disciplinas, el nexo fundante con la factualidad de los fenómenos sociales, el aprendizaje de las prácticas para constituir sujetos que actúen en la realidad con autonomía. Con esto queremos destacar que el campo de las ciencias sociales en la vida académica supone tres tipos de actividades que deben ser abordadas de manera simultánea: el desarrollo de pensamiento analítico, el desarrollo de capacidad investigativa empírica y el aprendizaje de 
las prácticas profesionales del caso. En la formación universitaria, el abordaje integrado de estos tres tipos de actividades supone una efectiva integración disciplinaria de la teoría y la práctica. Tal integración, en el caso de las ciencias sociales, se ve a veces dificultada por el empuje hacia la profesionalización, que tiende a manifestarse en la percepción de los estudiantes respecto de que la teoría y la práctica de sus disciplinas van por carriles separados en los planes de estudios a los que ellos deben ajustar su progresión académica.

Hay sin embargo un tema más de fondo en esta percepción de insuficiente o nula integración de teoría y práctica y ello tiene que ver con los cambios muy importantes que se están produciendo en los ámbitos disciplinarios y profesionales de las ciencias sociales. Al respecto se puede advertir dos movimientos que tienden a ser convergentes.

Por una parte una acentuada flexibilización de los perfiles ocupacionales de las profesiones, que se manifiesta en que las plazas laborales ocupadas por individuos que han sido entrenados en las profesiones más clásicas de las disciplinas, son cada vez más variadas en sus contenidos de destrezas pertinentes y en sus niveles jerárquicos en las organizaciones. Eso se traduce en trayectorias ocupacionales muy dinámicas, y dotadas de gran movilidad tanto vertical como horizontal. En este marco de flexibilización creciente, sin embargo, la formulación de los perfiles profesionales de las carreras no guarda necesariamente relación con el dinamismo ocupacional.

De allí entonces que una tarea urgente en las carreras profesionales de las ciencias sociales, es producir una completa reformulación de tales perfiles, centrando tal vez el núcleo de la formación en un paquete de destrezas generales básicas de las disciplinas implicadas, con muy alto niveles de exigencia en esos componentes, y dejando relativamente poco espacio para la especialización en el pregrado, lo cual implica necesariamente acortar substancialmente los componentes lectivos de las mallas curriculares. En síntesis, desde el punto de vista curricular, se trata de desescolarizar la formación de pregrado, para marcar radicalmente la transición desde la educación media pedagógicamente escolarizada a la educación superior que tiene que operar de otra manera, es decir, con una pedagogía que busca que el sujeto se adueñe de su propia formación y que tome decisiones para tal fin.

Por otra parte se observa una tendencia sostenida al debilitamiento de las fronteras disciplinarias, de modo que la educación superior tiene que hacerse cargo hoy de la marcada porosidad de los marcos analíticos centrales de las disciplinas, tanto en sus desarrollos propiamente teóricos como epistemológicos 
y metodológicos. Esto tiene importantes consecuencias para la introducción de dimensiones de multidisciplinariedad en las mallas curriculares y también de transdisciplinariedad si es que se lograse introducir de manera orgánica en las mallas y los planes de estudios algunos ejes integrados de problematización, ojalá en estrecha vinculación con la realidad y las tendencias de cambios que se han señalado ya en el inicio de este trabajo.

Después de todo, es probable que el mejor ejercicio de la autonomía de la persona, sea la capacidad adquirida de enfrentar y resolver problemas reales de su entorno que, por lo general, tienen causalidades múltiples. Allí se juega el desafío de la innovación curricular que es hoy una tarea ineludible en las instituciones de educación superior.

\section{REFERENCIAS BIBLIOGRÁFICAS.}

Atria, R., 2000

Atria, R., 2011

Barnett, R,. 1994

Cox, Cristian., 2000

Harvey, Lee y

Green, Diana, 1997
Calidad de la docencia de pregrado: reflexiones a partir de una experiencia personal, en Consejo Superior de Educación, Seminario Internacional 1997, Educación Superior: teoría y práctica de la docencia de pregrado, Santiago

"La situación de la investigación universitaria en Ciencias Sociales en Chile", en CPU, Revista Estudios Sociales, N¹19/2011, Santiago

The limits of competence. Knowledge, higher education and society, SRHE, Londres, Reino Unido.

Cambio curricular de la educación media e interrogantes para la educación superior, en Consejo Superior de Educación, Seminario Internacional 1997, Educación Superior: teoría y práctica de la docencia de pregrado, Santiago. Fuente original Barnet R, 1994

Defining quality. Assessment and Evaluation in Higher Education, An international journal, vol. 18 $\left(\mathrm{N}^{\circ} 1\right)$ 